\title{
Aortic calcification in patients with rheumatoid arthritis and ankylosing spondylitis: a cross- sectional case-control study
}

\section{Xuan Zhao}

Qingdao Women and Childrens Hospital

\section{Qian Wang}

Huashan Hospital Fudan University

\section{Yuanyuan Luo}

The affiliated Hospital of Qingdao University

\section{Yingshuang Liu}

The Affiliated Hospital of Qingdao University

\section{Song Gao}

The Affiliated Hospital of Qingdao University

\section{Wenbin Cong}

The Affiliated Hospital of Qingdao University

\section{Hua Li}

The Affiliated Hospital of Qingdao University

Hui Li

Qingdao Women and Childrens Hospital

Mingshu Sun ( $\nabla$ mingshu.sun@yahoo.com )

Affiliated Hospital of Qingdao University https://orcid.org/0000-0001-9671-5323

\section{Research article}

Keywords: Rheumatoid arthritis, Ankylosing spondylitis, Cardiovascular disease, Inflammation

Posted Date: April 7th, 2020

DOl: https://doi.org/10.21203/rs.3.rs-21064/v1

License: (c) (i) This work is licensed under a Creative Commons Attribution 4.0 International License. Read Full License 


\section{Abstract}

Objectives: Vascular calcification contributes to cardiovascular consequences in rheumatoid arthritis (RA) and ankylosing spondilytis (AS). This study was to evaluate the aortic calcification in RA and AS, and investigate possible risk factors.

Methods: The in-patients with RA or AS were consecutively recruited from a general hospital. Control subjects were healthy population recruited from the same hospital. The aortic calcification score (ACS) was obtained by 256-slice spiral CT scanners using the Heart Beat-CS program (Philips Medical Systems, Cleveland Ohio, USA).

Results: 146 RA patients, 97 AS patients and 200 controls were included. Serum triglyceride, cholesterol, urate, high and low density liptein cholesterols in RA or AS were all lower than that in controls. The ACS was higher in RA group than in AS and control groups. Only young ( $\leq 50 \mathrm{yrs}$ ) female AS had ACS higher than controls. Compared with the controls, RA increased the risk for aortic calcification by $4.72(95 \% \mathrm{Cl}$ 2.78-7.99) fold after adjusted for age and sex. While AS in general, did not. Advanced age, male sex and elevated blood glucose were the risk factors for ACS in controls; advanced age, high C-reactive protein (CRP) and more co-morbidities were the risk factors in AS; and advanced age, long disease course, high DAS28(CRP) were risk factors in RA.

Conclusions: Patients with RA or AS were more likely to develop aortic calcification compared with age and sex-matched healthy controls. Long disease course and high inflammatory criteria were risk factors, which advocate an intense control of disease associated systemic inflammation.

\section{Background}

Rheumatoid arthritis (RA) is a systemic autoimmune disease with chronic erosive arthritis and extraarticular manifestations like respiratory and neurologic involvement. Treating targets usually focus on the management of disease activity concerning arthritis and maintenance of joint functions. The outcome of arthritis has greatly improved as a benefit of this strategy in recent years [1]. While an Australian cohort study showed that RA patients had a life expectancy 6 to 10 years shorter than age-matched general population, with cardiovascular disease (CVD) being the leading cause of death [2]. Ankylosing spondylitis (AS) is also a chronic inflammatory disease mainly affecting the spinal joints. Up to $30 \%$ of the patients will suffer co-morbidities like anterior uveitis, aortitis, cardio conduction abnormalities and pericarditis. A population-based study indicated that the 10-year cumulative incidence of cardiovascular disease in AS patients was three times higher than predicted (standardized incidence ratio $3.01,95 \% \mathrm{Cl}$ 1.35-6.69) [3]. Other studies also revealed that the crude or age- and sex-adjusted mortalities were higher in AS patients than in general populations, and the major cause of death was CVD [4, 5].

Meanwhile, RA patients present earlier, more overwhelming and more severe vascular calcification compared with non-RA patients, regardless of the conventional cardiovascular risk factors [6-8]. Vascular calcification of large arteries such as the aorta will result in increased arterial stiffness, left 
ventricular hypertrophy and congenital heart failure. These are the common feature of CVD among several so called systemic inflammatory conditions, such as diabetes mellitus and chronic kidney disease [9]. Patients with RA or AS have similar inflammatory status and CVD characteristics with these diseases. The risk of non-ischemic heart failure increased rapidly after RA onset, in contrast to the risk of ischemic heart failure; and high disease activity was associated mostly with non-ischemic heart failure [10].

So far, studies addressing CVD in RA or AS focused mainly on coronary artery calcification and seldom on aorta calcification. In this case control study, we investigated aortic calcification score (ACS) in RA and AS patients, and try to find clinical factors associated with it. It may provide us useful knowledge about CVD in RA and AS, and help with better management strategy and overall outcome in these diseases.

\section{Subjects And Methods}

\section{Study subjects}

In this case-control study, patients with RA or AS were consecutively recruited from the Department of Rheumatology and Immunology, Affiliated Hospital of Qingdao University between June 2014 and October 2019. RA was diagnosed according to the 2010 American College of Rheumatology (ACR)/European League against Rheumatism (EULAR) classification criteria; and AS, the Modified New York Criteria. Control participants were healthy people recruited from the Health Examination Center of the same hospital in the same time period. Subjects with histories of smoking or drinking, diabetes, hypertension, cardiovascular disease, cancer, acute or chronic infectious disease, other autoimmune disease and liver or kidney dysfunction were excluded. This study was approved by the Ethics Committee of the Affiliated Hospital of Qingdao University, and all study participants provided written informed consent.

\section{Clinical and biochemical parameters}

Demographic and clinical data of each private patient were obtained from the electric health record, including the time of symptom experience and standardized therapy before enrollment, and the visual analogue score (VAS) of general health status. The tenderness and swollen of 28 joints were evaluated in patients with RA, Including the shoulders, elbow joints, wrists, knees and bilateral $1^{\text {st }}$ to $5^{\text {th }}$ metacarpophalangeal and proximal interphalangeal joints.

Erythrocyte sedimentation rate (ESR) was determined by sodium citrate anticoagulant and Wechsler method; C-reactive protein (CRP) was detected by a latex-enhanced turbidimetric assay using reagents and instrumentation provided by Goldsite (Shenzhen, China); total cholesterol (TC), triglyceride (TG), high density lipoprotein cholesterol (HDL-C), low density lipoprotein cholesterol (LDL-C), fasting blood glucose (FBG) and serum urate (SU), by enzyme colorimetric methods. Rheumatoid factor (RF) was detected by the rate scatter immunoturbidimetry (N Latex RF kit, Siemens Healthcare Diagnositics Products GmhH, Germany), anti-CCP antibody (ACPA) by electrochemiluminescence (Elecsys Anti-CCP, Roche diagnositics $\mathrm{GmbH}$, Germany), and human leukocyte antigen B27 (HLA-B27) by flow cytometry method (IOTest ${ }^{\circledR}$ HLA- 
B27 FITC/HLA-B7-PE, Immunotech S.A.S, France). The disease activity scores in the 28 joints with ESR or CRP (DAS28 (CRP/ESR)) were calculated according to the formula previously reported [11].

\section{ACS measurement}

The aortic calcification scores (ACS) (including ascending aorta, aorta arch and aorta thoracic) were obtained automatically by the heart cycle gated 256-slice spiral CT scanner (Philips Medical Systems, Cleveland Ohio, USA) with the Heart Beat-CS calcification score automatic analysis software [12]. Scanning parameters were $120 \mathrm{kV} 55 \mathrm{~mA}$, frame rotation time $0.42 \mathrm{~s}$, slice thickness $2.5 \mathrm{~mm}$, reconstruction interval $2.5 \mathrm{~mm}$. Scanning field would cover between the $2-3 \mathrm{~cm}$ above the lung tip and the costal diaphragmatic angle.

\section{Statistics}

Demographic and clinical features were summarized using standard descriptive statistics including mean (standard deviation), or median (interquartile range), frequency or percent as appropriate. Comparisons of variables between different groups were undertaken using independent t-test, Mann-Whitney $\mathrm{U}$ test, $\mathrm{c}^{2}$ tests, one-way ANOVA and Kruskal-Wallis tests as appropriate. Spearman correlation analyses were applied for understanding the relationships between clinical factors and ACS in each group. Multivariate logistic regression models were established to explore independent risk factors for aortic calcification within each group, and the impact of disease on aortic calcification. The aortic calcification was defined as ACS $>0$. Two-sided tests with a $5 \%$ significance level $(p<0.05)$ were considered statistically significant. All statistical analyses were performed using SPSS version 23.0 (IBM, Armonk, NY, USA).

\section{Results}

\section{Clinical features}

146 RA patients were included in the RA group, 97 in the AS group and 200 healthy anticipants in the control group (Table 1). There was no significant difference in age between RA, AS and the control group (53 (10) vs 52 (6) vs 51 (9) years, $\mathrm{p}=0.21$ ). There were more male patients in AS group than in RA group or in controls $(50 \%$ vs $30.8 \%$ vs $38.5 \%, p=0.01)$. Although the biochemical criteria were generally within normal ranges, the concentrations of TG, TC, LDL-C, HDL-C and SU were lower in RA and AS group than in control group $(p<0.005)$. Meanwhile the FBG level was lower in AS group compared with those in the other two groups $(\mathrm{p}=0.002)$. RA patients had higher CRP $(19.9(5.5-46.5)$ vs $6.2(1.5-16.7) \mathrm{mg} / \mathrm{L}, \mathrm{p}<$ $0.001)$ and ESR (31.7 (17.4-60.1) vs $15.8(8.4-30.1) \mathrm{mm} / \mathrm{h}, \mathrm{p}<0.001)$ levels, but less co-morbidities (0.0 $(0.0-0.0)$ vs $1.0(0.0-1.0), \mathrm{p}<0.001)$ than AS patients. 
Table 1

General information and disease-related parameters of all participants

\begin{tabular}{|c|c|c|c|c|c|}
\hline & RA $(n=146)$ & AS $(n=97)$ & $\begin{array}{l}\text { Control }(n= \\
200)\end{array}$ & $\begin{array}{l}\text { Statistic } \\
\text { Values }\end{array}$ & $\mathbf{P}$ \\
\hline \multicolumn{6}{|c|}{ General information } \\
\hline Age (yrs) & $53(10)$ & $52(6)$ & $51(9)$ & 3.152 & 0.207 \\
\hline Male sex, n (\%) & $45(30.8)$ & $48(50)^{\#}$ & $77(38.5)$ & 8.566 & 0.014 \\
\hline TG (mmol/L) & $0.9(0.6-1.3)^{*}$ & $0.9(0.6-1.1)^{*}$ & $1.0(0.7-1.4)$ & 14.001 & 0.001 \\
\hline TC (mmol/L) & $4.3(1.1)^{\star}$ & $4.1(0.7)^{\star}$ & $4.9(0.9)$ & 61.622 & 0.000 \\
\hline $\mathrm{HDL}-\mathrm{C}(\mathrm{mg} / \mathrm{dL})$ & $1.2(0.9-1.4)^{\star}$ & $1.2(1.1-1.4)^{\star}$ & $1.5(1.3-1.7)$ & 78.917 & 0.000 \\
\hline LDL-C (mg/dL) & $2.6(0.8) *$ & $2.5(0.6)^{\star}$ & $2.8(0.7)$ & 15.405 & 0.000 \\
\hline $\mathrm{FBG}(\mathrm{mmol} / \mathrm{L})$ & $4.8(4.3-5.1)$ & $4.5(4.2-4.9)^{\star \#}$ & $4.8(4.4-5.2)$ & 12.835 & 0.002 \\
\hline $\mathrm{SU}(\mu \mathrm{mol} / \mathrm{L})$ & $\begin{array}{l}210.8(175.3- \\
259.1)^{\star}\end{array}$ & $\begin{array}{l}244.1(213.0- \\
299.3)^{\star \#}\end{array}$ & $\begin{array}{l}276.3(231.5- \\
319.1)\end{array}$ & 63.033 & 0.000 \\
\hline \multicolumn{6}{|c|}{ Disease-related parameters } \\
\hline $\begin{array}{l}\text { Disease course } \\
\text { (yrs) }\end{array}$ & $5.0(1.5-10.0)$ & $8.0(2.0-20.0)^{\#}$ & & 5641.5 & 0.007 \\
\hline CRP (mg/L) & $19.9(5.5-46.5)$ & $6.2(1.5-16.7)^{\#}$ & & 4145.5 & 0.000 \\
\hline $\operatorname{ESR}(\mathrm{mm} / 1 \mathrm{~h})$ & $\begin{array}{l}31.7(17.4- \\
60.1)\end{array}$ & $15.8(8.4-30.1)^{\#}$ & & 4386.0 & 0.000 \\
\hline DAS28 (CRP) & $4.9(1.2)$ & - & & & \\
\hline DAS28 (ESR) & $5.3(1.3)$ & - & & & \\
\hline $\mathrm{RF}(\mathrm{IU} / \mathrm{mL})$ & $\begin{array}{l}79.8(18.9- \\
353.6)\end{array}$ & - & & & \\
\hline ACPA $(\mathrm{U} / \mathrm{mL})$ & $\begin{array}{l}341.3(114.1- \\
500.0)\end{array}$ & - & & & \\
\hline HLA-B27(\%) & - & $99.2(96.6-99.7)$ & & & \\
\hline $\begin{array}{l}{ }^{\&} \text { Co-morbidities } \\
\text { (n) }\end{array}$ & $0.0(0.0-0.0)$ & $1.0(0.0-1.0)^{\#}$ & & 3613.0 & 0.000 \\
\hline \multicolumn{6}{|c|}{$\begin{array}{l}\text { Data given as mean (standard deviation) or median (interquartile range); }{ }^{\&} \text { Including rheumatoid } \\
\text { nodules, respiratory, neurologic and eye involvements and vasculitis in RA, and uveitis, inflammatory } \\
\text { bowel disease, skin involvement, cardio transduction abnormalities and peripheral arthritis in AS; } \\
{ }^{\star} \text { Compared with control group, } p<0.05 \text {; }{ }^{*} \text { Compared with RA group, } p<0.05\end{array}$} \\
\hline
\end{tabular}


Aortic calcification score (ACS) between RA, AS and the control group

The general ACS was higher in RA group (19.4 (0.0-191.0)) than in AS group (0.0 (0.0-28.4)) and in controls $(0.0(0.0-13.8))(p<0.001)$. No difference of general ACS levels between AS group and the controls was observed ( $p>0.05)$. When stratified by sex and age ( $\leq$ or $>50$ years), RA patients in all subgroups and only female AS patients with age $\leq 50$ years had higher ACSs than sex and age-matched controls. Logistic regression analysis revealed that RA was associated with 3.77 (95\% Cl 2.37-5.99) fold greater relative odds of aortic calcification than controls, and $4.72(95 \% \mathrm{Cl} 2.78-7.99)$ fold after adjustment for age and sex, while AS did not increase the risk of aortic calcification $(O R=0.85,95 \% \mathrm{Cl}$ $\left.0.52-1.40 ; \mathrm{OR}_{\text {adjusted }}=0.75,95 \% \mathrm{Cl}(0.44,1.27)\right)$. These results are shown in Table 2 .

Table 2

Aortic calcification score (ACS) between RA, AS and control groups

\begin{tabular}{|c|c|c|c|c|c|}
\hline & RA $(n=146)$ & AS $(n=97)$ & $\begin{array}{l}\text { Control }(n= \\
200)\end{array}$ & $\begin{array}{l}\text { Statistic } \\
\text { Values }\end{array}$ & $\mathbf{P}$ \\
\hline \multicolumn{6}{|l|}{ ACS } \\
\hline Total & $19.4(0.0-191.0)^{\star}$ & $0.0(0.0-28.4)^{\#}$ & $0.0(0.0-13.8)$ & 58.678 & 0.000 \\
\hline Male, $\leq 50$ Years & $\begin{array}{l}19.1(18.7- \\
20.1)^{\star}\end{array}$ & $0.0(0.0-4.5){ }^{\#}$ & $0.0(0.0-18.2)$ & 29.703 & 0.019 \\
\hline Male, > 50 Years & $\begin{array}{l}183.2(38.1- \\
623.5)^{\star}\end{array}$ & $0.0(0.0-39.7){ }^{\#}$ & $2.2(0.0-63.6)$ & 29.703 & 0.000 \\
\hline $\begin{array}{l}\text { Female, } \leq 50 \\
\text { Years }\end{array}$ & $3.1(0.0-7.0)^{\star}$ & $0.0(0.0-32.1)^{\star}$ & $0.0(0.0-0.0)$ & 20.936 & 0.000 \\
\hline $\begin{array}{l}\text { Female, > } 50 \\
\text { Years }\end{array}$ & $\begin{array}{l}39.2(4.6- \\
262.0)^{\star}\end{array}$ & $2.8(0.0-70.9){ }^{\#}$ & $0.0(0.0-13.6)$ & 30.177 & 0.000 \\
\hline \multicolumn{6}{|c|}{ OR $(95 \% \mathrm{Cl})$ for aortic calcification $(\mathrm{ACS}>0)$} \\
\hline Unadjusted & $3.77(2.37-5.99)$ & $\begin{array}{l}0.85(0.52- \\
1.40)\end{array}$ & 1 & & \\
\hline \&Adjusted & $4.72(2.78-7.99)$ & $\begin{array}{l}0.75(0.44- \\
1.27)\end{array}$ & 1 & & \\
\hline
\end{tabular}

\section{Simple linear correlation analyses of ACS with clinical features within each group}

In control group, correlation analysis (Table 3 ) showed that the ACS was positively correlated with age, male sex, TG, LDL-C and FBG $(r=0.341,0.246,0.189,0.175$ and $0.202, p<0.05$, respectively), and negatively correlated with HDL-C $(r=-0.146, p=0.039)$, but no significant correlation with TC and SU $(p>$ 
0.05). In RA group, the ACS was positively correlated with age, male sex, TG, TC and LDL-C $(r=0.621$, $0.351,0.180,0.170$ and $0.195, p<0.05$, respectively), but not significantly correlated with HDL-C, FBG and SU ( $p>0.05)$. In addition, ACS in RA group was also positively correlated with the course of disease, CRP, DAS28 (CRP) and co-morbidities $(r=0.212,0.166,0.179$ and $0.316, p<0.05)$, but not significant correlated with ESR, DAS28 (ESR), RF, ACPA and time of therapy $(p>0.05)$. In AS group, ACS was positively correlated with age, CRP, ESR and co-morbidities $(r=0.243,0.234,0.216$ and $0.398, p<0.05)$, but not with disease course, TG, TC, HDL-C, LDL-C, FBG or SU ( $p>0.05)$.

Table 3

Simple linear correlation analysis of aortic calcification scores (ACS) with general information and disease-related parameters in each group

\begin{tabular}{|c|c|c|c|c|c|c|}
\hline & \multicolumn{2}{|c|}{ RA group } & \multicolumn{2}{|c|}{ AS group } & \multicolumn{2}{|c|}{ Control group } \\
\hline & $\mathbf{r}$ & $\mathbf{P}$ & $\mathbf{r}$ & $P$ & $r$ & $\mathbf{P}$ \\
\hline Age & 0.621 & 0.000 & 0.243 & 0.016 & 0.341 & 0.000 \\
\hline Male sex & 0.351 & 0.000 & -0.166 & 0.105 & 0.246 & 0.000 \\
\hline TG & 0.180 & 0.030 & -0.073 & 0.475 & 0.189 & 0.007 \\
\hline $\mathrm{TC}$ & 0.170 & 0.040 & -0.151 & 0.141 & 0.125 & 0.077 \\
\hline HDL-C & -0.044 & 0.600 & -0.020 & 0.844 & -0.146 & 0.039 \\
\hline LDL-C & 0.195 & 0.018 & -0.120 & 0.241 & 0.175 & 0.013 \\
\hline FBG & 0.089 & 0.286 & 0.052 & 0.616 & 0.202 & 0.004 \\
\hline SU & 0.143 & 0.085 & -0.196 & 0.054 & 0.08 & 0.260 \\
\hline Disease course & 0.212 & 0.010 & -0.014 & 0.893 & & \\
\hline CRP & 0.166 & 0.045 & 0.234 & 0.021 & & \\
\hline ESR & -0.043 & 0.606 & 0.216 & 0.033 & & \\
\hline DAS28 (CRP) & 0.179 & 0.030 & & & & \\
\hline DAS28 (ESR) & 0.082 & 0.325 & & & & \\
\hline RF & 0.070 & 0.404 & & & & \\
\hline ACPA & -0.095 & 0.255 & & & & \\
\hline HLA-B27 & & & -0.074 & 0.487 & & \\
\hline Co-morbidities & 0.316 & 0.000 & 0.398 & 0.000 & & \\
\hline Time of therapy & 0.074 & 0.377 & & & & \\
\hline
\end{tabular}


Logistic regression analysis (Figure) showed that the in the control group, risk factors for aortic calcification included advanced age $(\mathrm{OR}=1.11,95 \% \mathrm{Cl} 1.07-1.16)$, male sex $(\mathrm{OR}=2.59,95 \% \mathrm{Cl} 1.33-$ 5.04) and $F B G$ increasing $(O R=1.98,95 \% \mathrm{Cl} 1.14-3.42)$; in RA group, the risk factors included advanced age $(\mathrm{OR}=1.07,95 \% \mathrm{Cl} 1.02-1.12)$, long disease course $(\mathrm{OR}=1.22,95 \% \mathrm{Cl} 1.09-1.38)$ and high DAS28 (CRP) $(\mathrm{OR}=1.58,95 \% \mathrm{Cl} 1.08-2.31)$; in AS group, the risk factors included advanced age (OR $=1.09,95 \%$ $\mathrm{Cl} 1.01-1.18)$ ), high $\mathrm{CRP}(\mathrm{OR}=1.05,95 \% \mathrm{Cl} 1.00-1.10)$ and more co-morbidities $(\mathrm{OR}=2.80,95 \% \mathrm{Cl} 1.29-$ 6.11).

\section{Discussion}

Vascular calcification is a pathological process of calcium and phosphate salts accumulation within the arterial wall. It involves mainly two forms, intimal calcification in atherosclerosis and cardiac valve calcification, and artery tunica media calcification (also known as Mönckeberg sclerosis) in aging, diabetes mellitus, and chronic kidney disease mediated calcification, with various degrees overlap of them $[13,14]$. Vascular medial calcification increases vascular wall hardness and reducing vascular compliance. Aortic medial calcification induces cardiac pressure overload, leading to cardiovascular events such as congestive heart failure. According to previous studies [2-4], CVD was the leading cause of mortality in both RA and AS patients. The cardiovascular lesions in RA patients are characterized by earlier and more severe arterial calcification, as well as increased incidence and mortality of non-ischemic heart failure but not the incidence of atheromatous plaque $[8,10]$. AS patients also manifest accelerated atherosclerosis, arterial stiffness and myocardial infarction after adjustment of traditional CVD risk factors $[15,16]$. These data applaud that vascular calcification, especially aortic calcification may be an underlying mechanism of CVD in RA and AS patients.

This study evaluated the aortic calcification profiles in RA, AS patients and healthy controls, using a standard computerized tomography calcification score of the ascending aorta, aortic arch and thoracic aorta. The results showed that the aortic calcification scores (ACS) were elevated in all subgroups of RA patients than in sex and age-matched healthy controls. As if for AS, only females with age $\leq 50$ years had higher ACS level. Meanwhile, all subgroups of AS patients had lower ACSs than RA patients, except for the female $\leq 50$ years subgroup. RA increased the risk of aortic calcification by $4.72(95 \% \mathrm{Cl} 2.78-7.99)$ fold after adjustment for age and sex, while AS generally did not (Table 2). Thus, RA patients had more severe aortic calcification than AS patients and controls, especially male and female in advanced age; only young female AS patients had aortic calcification level comparable with RA patients and higher than controls. These results support the previous clinical epidemiologic studies $[2,10,15]$.

We further explored possible determinants for aortic calcification in all participants. Logistic regression analysis showed that only advanced age, male sex and FBG were independent risk factors for aortic calcification in healthy controls (Figure). Unlikely in studies concerning atherosclerosis [17], dislipidemia defined as high level of LDL-C, serum triglyceride and low level of HDL-C was not associated risk factor for aortic calcification in the present study. This implies a pathogenesis of calcification diversely from atherosclerosis. Although male sex had more severe coronary artery calcification score as reported by a 
Korean study [18], no convincing explanations available so far. Aging attributes to vascular calcification through multifaceted mechanisms [19]. Several researchers reported similar results that advanced age and FBG were independently related to aortic calcification in non-rheumatic population [20-23]. The common explanation underlies aging and diabetes is systemic inflammation $[9,24]$..

Advanced age as a risk factor for aortic calcification was consistent in RA and AS patients, as it was in controls. AS patients had lower level of FBG, which may be a confounding factor for the ACS in this group. While other traditional factors like TG, LDL-C, FBG and SU were generally decreased and not involved in aortic calcification in either RA or AS patients. These data indicate that disease associated factors participate in developing arterial calcification in these patients. Disease duration and DAS28 (CRP) were impact factors for ACS in RA patients, and CRP and co-morbidities in AS patients. All these parameters are criteria for disease activity related systemic inflammation. Therefore, our study implies that chronic systemic inflammation may be the main contributors for aortic calcification in these patients. Recent studies have established that inflammatory joint diseases are associated with an increased risk of CVD development related in part to the chronic systemic inflammation, which is often marked in RA and usually moderate in AS [25]. Female AS patients had a higher CVD risk, with an OR of 5.75 in females and 2.85 in males $[26,27]$. Our data are completely consistent with these research and might provide the pathological explanation for them.

RA and AS are all chronic inflammatory diseases that may affect multiple systems of the body, including the vascular bed. A recent prospective study reported that women with RA had increased risks of total and CVD mortality compared with those without RA (HR $=1.40$ and 1.45 , respectively) [28]. While another study from Netherlands showed that patients of early-onset RA under tight controlled treatment did not [29]. A cohort study revealed a doubled incidence of heart failure and an increased risk of mortality attributable to heart failure in patients with RA, with the risk of heart failure associated with RF positivity, repeatedly high ESR, severe extra-articular manifestations and corticosteroid use, and a protective effect against heart failure with methotrexate use [30]. Although data are limited, studies have confirmed that low disease activity of AS did not promote vascular calcification, and inflammatory related indicators were associated with increased mortality in patients with AS $[13,14]$. Taking together, all these data suggested that the clinical markers of high systemic inflammation level confer an additional risk for CVD among these patients.

Rho et al further reported that vascular calcification did not associated with Framingham risk scores, nor the existence of diabetes mellitus; and only IL-6 and TNFa were independent risk factors for coronary artery calcification in RA patients [31]. IL-6 and TNFa are the key inflammatory cytokines indentified in RA and AS. IL-6 is a biopleiotropic cytokine and can induce multiple systematic effects, like hypoalbuminemia, anemia, apoptosis, weakness, dyslipidemia and the production of CRP in hepatocytes. CRP is closely related to inflammatory activity, CVD and other co-morbidities in RA [32,33]. More and more studies including ours have shown that patients with RA and AS tend to have low TC and LDL-C levels, as well as low HDL-C level [34-36]. They found that there was a negative correlation between CRP and HDL-C levels in active RA and AS, which was consistent with the results of this study $(r=-0.262, P=$ 
0.001 in $R A ; r=-0.270, P=0.007$ in AS). Thus, our study also showed a systemic inflammatory profile prominently labeled by serum CRP levels in both RA and AS patients. Meanwhile, evidences of the relationship between IL-6 and vascular calcification are spring up [37]. Co-morbidities reflect the degree of target organ involvement of the disease. $53.2 \%$ of RA patients with high disease activity were complicated with other conditions at the disease onset, of which $23 \%$ developed other co-morbidities during followed up [38, 39]. This study found that the co-morbidity frequency tended to be a contributor to vascular calcifications in those patients (with $\mathrm{OR}=2.88,95 \% \mathrm{Cl} 0.95-8.74$ in $\mathrm{RA}$, and $\mathrm{OR}=2.80,95 \% \mathrm{Cl}$ 1.29-6.11 in AS), which was consistent with the previous studies. Aortic calcification induced mainly by systemic inflammation might be the key problem and the most important contributor to cardiovascular death in RA and AS.

We didn't find any relationships between ACS and other disease related criteria, like RF and ACPA in RA, or HLA-B27 in AS. Apparently, RF, ACPA and HLA-B27 are more likely immunological disorders contributing to disease initiation rather than tissue inflammation [40]. Serum concentration of RF and ACPA will change the ESR level and made it not well consistent with CRP, which is a well-established marker of inflammation. This explained our results that ESR was in dependent with CRP and correlated with ACS in AS, but not in RA patients.

This pilot study has several limitations. Firstly, because this is a cross-sectional study, it's hard to draw a causal conclusion of systemic inflammation on the aortic calcification. Longitudinal studies and research addressing the underlying mechanisms are needed. Secondly, although this study suggests aortic calcification score as a marker for systemic inflammation, we cannot build the normal range of ACS in age and sex-matched healthy controls given the insufficient sample size. The third, detailed changes of the cardiovascular system should be profiled to provide more clinical information, as well as the cytokine spectrum.

\section{Conclusion}

This study highlights the role of ACS as the marker indicating long-term systemic inflammation in RA and AS patients. In addition, 256 slice spiral CT is commonly used in clinic, which can provide a reliable and reproducible method for the examination of aortic calcification. It also showed that there was no significant correlation between treatment time and ACS in RA patients, implying incomplete disease control and yielding for improving the treatment strategy in this group. More attentions should be paid to a complete disease evaluation including cardiovascular system in these patients. Further studies are called on to investigate this phenomenon for the sake of a better prognosis in these patients.

\section{Abbreviations}

RA, rheumatoid arthritis; AS, ankylosing spondylitis; TG, triglyceride; TC, total cholesterol; HDL-c, highdensity lipoprotein cholesterol; LDL-C, low-density lipoprotein cholesterol; FBG, fasting blood glucose; SU, serum urate; CRP, C-reactive protein; ESR, erythrocyte sedimentation rate; DAS28, disease activity score in 
the 28 joints; RF, rheumatoid arthritis; ACPA, anti-citrullinated peptide antibody; HLA, human leukocyte antigen; OR, odds ratio; $95 \% \mathrm{Cl}, 95 \%$ confidence interval

\section{Declarations}

\section{Acknowledgement}

Not applicable.

\section{Authors' contributions}

M.S. takes full responsibility for the work, had access to the data and controlled the decision to publish. M.S. conceived of the study, developed the protocol and completed data interpretation and the manuscript revision. S.G. and W.C. analysed the CT scans. Hua L. and Hui L. coordinated the study. Y. Liu. and X.Z. assisted with the participant recruitment and study visits. X.Z., Q.W. and Y. Luo did the data obtaining and statistical analyses. X.Z. drafted the the manuscript. All authors contributed to manuscript revisions and approved the final manuscript.

\section{Funding}

This study was supported by the research projects grants from the National Natural Science Foundation of China (\#81871288) and the Natural Science Foundation of Shandong Province (\#ZR2018MH015).

\section{Availability of data and materials}

The datasets used and analyzed during the current study are available from the corresponding author on reasonable request.

\section{Ethics approval and consent to participate}

The study was approved by the ethics committee of Affiliated Hospital of Qingdao University, and informed consents were obtained from all patients.

\section{Consent for publication}

Not applicable.

\section{Competing interests}

All authors declare that they have no competing of interest with regard to the work.

\section{References}

1. Smolen JS, Aletaha D, Barton A, et al. Rheumatoid arthritis. Nat Rev Dis Primers. 2018;4:18001. 
2. Lassere MN, Rappo J, Portek IJ, Sturgess A, Edmonds JP. How many life years are lost in patients with rheumatoid arthritis? Secular cause-specific and all-cause mortality in rheumatoid arthritis, and their predictors in a long-term Australian cohort study. Intern Med J. 2013;43(1):66-72.

3. Wright KA, Crowson CS, Michet CJ, Matteson EL. Time Trends in Incidence, Clinical Features, and Cardiovascular Disease in Ankylosing Spondylitis Over Three Decades: A Population-Based Study. Arthritis Care Res (Hoboken). 2015;67(6):836-41.

4. Exarchou $\mathrm{S}$, Lie E, Lindstrom $\mathrm{U}$, et al. Mortality in ankylosing spondylitis: results from a nationwide population-based study. Ann Rheum Dis. 2016;75(8):1466-72.

5. Buschiazzo EA, Schneeberger EE, Sommerfleck FA, Ledesma C, Citera G. Mortality in patients with ankylosing spondylitis in Argentina. Clin Rheumatol. 2016;35(9):2229-33.

6. Giles JT, Szklo M, Post W, et al. Coronary arterial calcification in rheumatoid arthritis: comparison with the Multi-Ethnic Study of Atherosclerosis. Arthritis Res Ther. 2009;11(2):R36.

7. Chung CP, Oeser A, Raggi P, et al. Increased coronary-artery atherosclerosis in rheumatoid arthritis: Relationship to disease duration and cardiovascular risk factors. Arthritis Rheum. 2005;52(10):304553.

8. Wang S, Yiu KH, Mok MY, et al. Prevalence and extent of calcification over aorta, coronary and carotid arteries in patients with rheumatoid arthritis. J Intern Med. 2009;266(5):445-52.

9. Chen NX, Moe SM. Pathophysiology of Vascular Calcification. Curr Osteoporos Rep. 2015;13(6):37280.

10. Mantel A, Holmqvist M, Andersson DC, Lund LH, Askling J. Association Between Rheumatoid Arthritis and Risk of Ischemic and Nonischemic Heart Failure. J Am Coll Cardiol. 2017;69(10):1275-85.

11. van der Heijde DM, van 't Hof MA, van Riel PL, et al. Judging disease activity in clinical practice in rheumatoid arthritis: first step in the development of a disease activity score. Ann. Rheum Dis. 1990;49(11):916-20.

12. An C, Lee HJ, Lee HS, et al. CT-based abdominal aortic calcification score as a surrogate marker for predicting the presence of asymptomatic coronary artery disease. Eur Radiol. 2014;24(10):2491-8.

13. O'Rourke C, Shelton G, Hutcheson JD, et al. Calcification of Vascular Smooth Muscle Cells and Imaging of Aortic Calcification and Inflammation. J Vis Exp. 2016;111.

14. Kwon D, Kim Y, Kook H. New Aspects of Vascular Calcification: Histone Deacetylases and Beyond. J Korean Med Sci. 2017;32(11):1738-48.

15. Mathieu S, Gossec L, Dougados M, Soubrier M. Cardiovascular profile in ankylosing spondylitis: a systematic review and meta-analysis. Arthritis Care Res (Hoboken). 2011;63(4):557-63.

16. Peters MJ, van Eijk IC, Smulders YM, et al. Signs of accelerated preclinical atherosclerosis in patients with ankylosing spondylitis. J Rheumatol. 2010;37(1):161-6.

17. Ma YH, Leng XY, Dong Y, et al. Risk factors for intracranial atherosclerosis: A systemic review and meta-analysis. Atherosclerosis. 2019;281:71-7. 
18. Lee DH, Youn HJ, Yi JE, et al. Sex difference in osteoporosis and vascular calcification associated with age. Korean Circ J. 2013;43(7):453-61.

19. Pescatore LA, Gamarra LF, Liberman M. Multifaceted mechanisms of vascular calcification in ageing. Arterioscler Thromb Vasc Biol. 2019;39(7):1307-16.

20. Rutsch F, Nitschke Y, Terkeltaub R. Genetics in Arterial Calcification. Circ Res. 2011;109(5):578-92.

21. England BR, Thiele GM, Anderson DR, Mikuls TR. Increased cardiovascular risk in rheumatoid arthritis: mechanisms and implications. BMJ. 2018;361:k1036.

22. Arida A, Protogerou A, Kitas G, Sfikakis P. Systemic Inflammatory Response and Atherosclerosis: The Paradigm of Chronic Inflammatory Rheumatic Diseases. Int J Mol Sci. 2018;19(7):1890.

23. Wu M, Rementer C, Giachelli CM. Vascular Calcification: An Update on Mechanisms and Challenges in Treatment. Calcif Tissue Int. 2013;93(4):365-73.

24. Durham AL, Speer MY, Scatena M, Giachelli CM, Shanahan CM. Role of smooth muscle cells in vascular calcification: implications in atherosclerosis and arterial stiffness. Cardiovasc Res. 2018;114(4):590-600.

25. Mason JC, Libby P. Cardiovascular disease in patients with chronic inflammation: mechanisms underlying premature cardiovascular events in rheumatologic conditions. Eur Heart J. 2015;36(8):482-9c.

26. Peters MJ, van der Horst-Bruinsms IE, Dijkmans BA, Nurmohamed MT. Cardiovascular risk profile of patients with spondylarthropathies, particularly ankylosing spondylitis and psoriatic arthritis. Semin Arthritis Rheum. 2004;34(3):585-92.

27. Lin CW, Huang YP, Chiu YH, Ho YT, Pan SL. Increased risk of ischemic stroke in young patients with ankylosing spondylitis: a population-based longitudinal follow-up study. PLoS One. 2014;9(4):94027.

28. Sparks JA, Chang SC, Liao KP, et al. Rheumatoid Arthritis and Mortality Among Women During 36 Years of Prospective Follow-Up: Results From the Nurses' Health Study. Arthritis Care Res (Hoboken). 2016;68(6):753-62.

29. Markusse IM, Akdemir G, Dirven L, et al. Long-Term Outcomes of Patients With Recent-Onset Rheumatoid Arthritis After 10 Years of Tight Controlled Treatment: A Randomized Trial. Ann Intern Med. 2016;164(8):523-31.

30. Myasoedova E, Crowson CS, Nicola PJ, et al. The influence of rheumatoid arthritis disease characteristics on heart failure. J Rheumatol. 2011;38(8):1601-6.

31. Rho YH, Chung CP, Oeser A, et al. Inflammatory mediators and premature coronary atherosclerosis in rheumatoid arthritis. Arthritis Rheum. 2009;61(11):1580-5.

32. Solomon DH, Kremer J, Curtis JR, et al. Explaining the cardiovascular risk associated with rheumatoid arthritis: traditional risk factors versus markers of rheumatoid arthritis severity. Ann Rheum Dis. 2010;69(11):1920-5.

33. Arida A, Protogerou AD, Konstantonis G, et al. Subclinical Atherosclerosis Is Not Accelerated in Patients with Ankylosing Spondylitis with Low Disease Activity: New Data and Metaanalysis of 
Published Studies. J Rheumatol. 2015;42(11):2098-105.

34. Bakland G, Gran JT, Nossent JC. Increased mortality in ankylosing spondylitis is related to disease activity. Ann Rheum Dis. 2011;70(11):1921-25.

35. Danielsen R, Sigvaldason H, Thorgeirsson G, Sigfusson N. Predominance of aortic calcification as an atherosclerotic manifestation in women: the Reykjavik study. J Clin Epidemiol. 1996;49(3):383-7.

36. Tsai JP, Kuo R, Sun JY, et al. Thoracic aortic calcification across the clinical dysglycemic continuum in a large Asian population free of cardiovascular symptoms. PLoS One. 2019;14(1): e0207089.

37. Hartman J, Frishman WH. Inflammation and Atherosclerosis. Cardiol Rev. 2014;22(3):147-51.

38. New SEP, Aikawa E. Cardiovascular Calcification. Circ JI. 2011;75(6):1305-13.

39. Rajamannan NM. Mechanisms of aortic valve calcification: the LDL-density-radius theory: a translation from cell signaling to physiology. Am J Physiol Heart Circ Physiol 2010;298(1):H5-H15.

40. Wigerblad G, Bas DB, Fernades-Cerqueira $C$, et al. Autoantibodies to citrullinated proteins may induce joint pain independent of inflammation. Ann Rheum Dis. 2016;75(4):730-8.

\section{Figures}

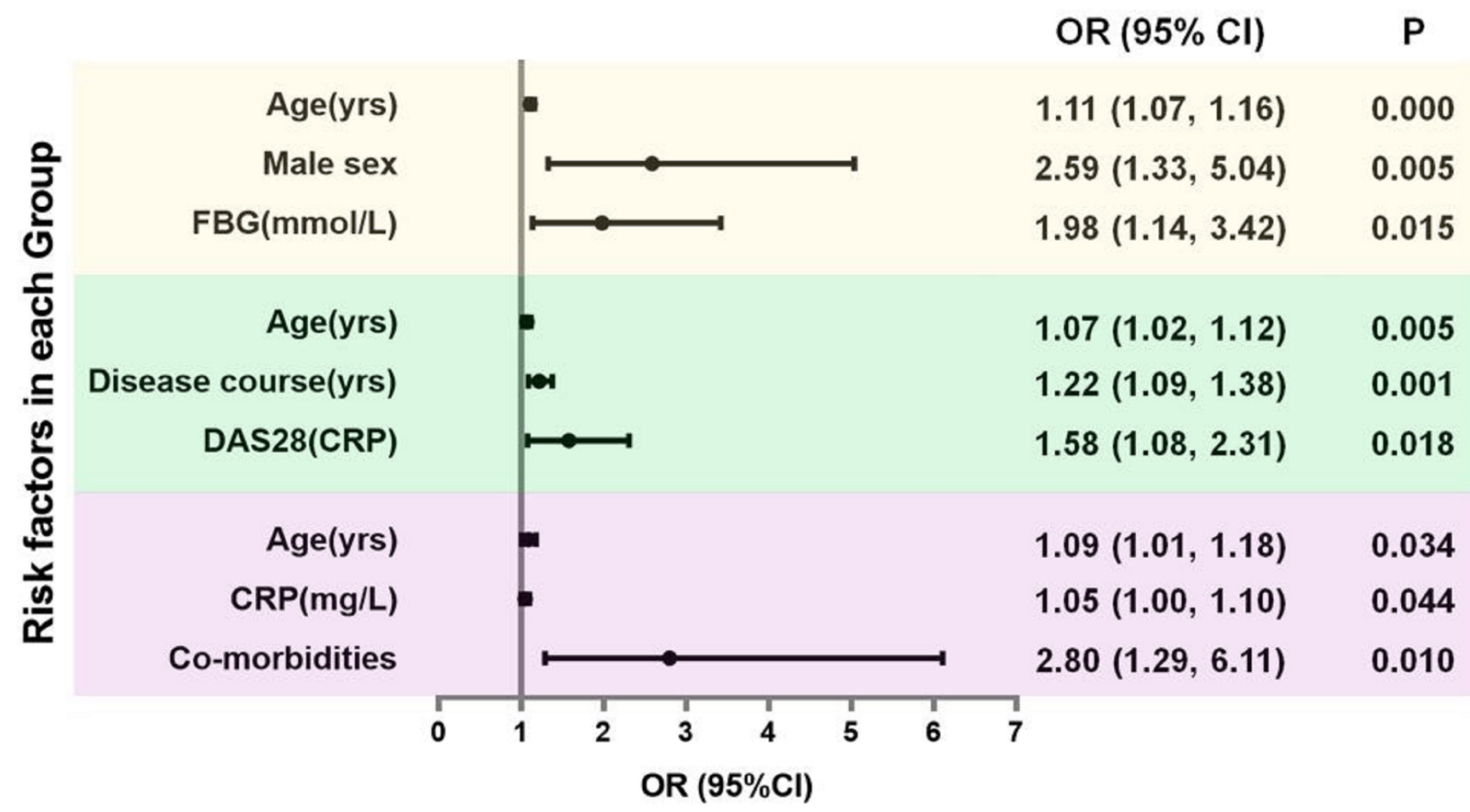

r-I Control group

-H RA group

HAS group

Figure 1 
Impact factors for aortic calcification (ACS $>0$ ) in each group. The data was analyzed by logistic regression multivariate analysis. The $\mathrm{OR}$ values indicate the risk to develop aortic calcification for each unit additional increase on the covariate. 